\title{
Endovascular fenestration in aortic dissection with acute malperfusion syndrome: Immediate and late follow-up
}

\author{
Marco Midulla, MD, ${ }^{\text {a }}$ Armelle Renaud, MD, ${ }^{\mathrm{a}}$ Thomas Martinelli, MD, ${ }^{\mathrm{a}}$ Mohammad Koussa, MD, \\ Claire Mounier-Vehier, MD, $\mathrm{PhD},{ }^{\mathrm{c}}$ Alain Prat, $\mathrm{MD},{ }^{\mathrm{d}}$ and Jean-Paul Beregi, MD, $\mathrm{PhD}^{\mathrm{a}}$
}

Objective: To evaluate the immediate and long-term results of fenestration in aortic dissection with acute malperfusion syndrome.

\begin{abstract}
Methods: Between 1999 and 2007, 35 patients (31 men; age, $57 \pm 11$ years) with aortic dissection (19 with type A and 16 with type B) were treated by fenestration for malperfusion syndrome ( 27 renal, 27 bowel, and 14 lower limb) due to dynamic compression. Fenestration was performed with 2 rigid guidewires inserted in one $8 \mathrm{~F}$ sheath ( $45 \mathrm{~cm}$ long). The mean interval between aortic dissection and fenestration was 5 days ( 28 patients within 3 days and 7 patients between 9 and 41 days).
\end{abstract}

Results: Fenestration (100\% technical success rate) with an additional endovascular procedure (29 peripheral stents and 1 thoracic stent graft) resulted in angiographic success in $97 \%$ of the patients. Bowel surgery was performed in 7 patients. Mortality within the first month (12/35) was related to bowel ischemia $(n=5)$, neurologic complications $(n=3)$, type A surgery $(n=2)$, and late treatment $(n=2)$. At a mean follow-up of $48 \pm 30$ months, 4 of the remaining 23 patients had died and 2 had withdrawn from the study. The diameter of the aorta, as measured using computed tomography/magnetic resonance imaging, remained stable in 12 of the remaining 17 patients and had increased in 5 ( 1 with Marfan syndrome and 4 with multiple arterial ectasia).

Conclusions: In emergencies, fenestration saved $69 \%$ of the patients with acute malperfusion syndrome in complicated aortic dissection. During the follow-up period, the aortic diameter remained stable in most of the surviving patients. (J Thorac Cardiovasc Surg 2011;142:66-72)

Video clip is available online.

Aortic dissection is rare $(1 \%)$ but has been associated with high mortality ( $50 \%$ within 48 hours). ${ }^{1}$ The high rates of mortality and morbidity ${ }^{2-4}$ have resulted from aortic rupture or extension of the longitudinal aortic dissection into the pericardium, aortic valve, or branches (coronary arteries, supra-aortic trunks, visceral arteries, lower limbs, or lumbar arteries). Many studies ${ }^{5-8}$ have focused on the place of cardiac surgery. In cases of type A aortic dissection, surgical replacement of the ascending aorta is obligatory to treat or prevent cardiac complications. In type B aortic dissection, medical treatment has been the

From the Departments of CardioVascular Imaging and Intervention, ${ }^{\mathrm{a}}$ CardioVascular Surgery, ${ }^{\text {b }}$ Vascular Medicine, ${ }^{\mathrm{c}}$ and Cardiac Surgery, ${ }^{\mathrm{d}}$ Hôpital Cardiologique, CHRU de Lille, France.

Disclosures: Authors have nothing to disclose with regard to commercial support. Received for publication Jan 28, 2010; revisions received June 22, 2010; accepted for publication July 31, 2010.

Address for reprints: Jean-Paul Beregi, MD, PhD, Department of CardioVasculaire Imaging and Intervention, Hôpital Cardiologique, CHRU de Lille 59037 Lille Cedex, France (E-mail: jean-paul.beregi@ chru-lille.fr).

$0022-5223 / \$ 36.00$

Copyright (c) 2011 by The American Association for Thoracic Surgery doi: $10.1016 /$ j.jtcvs.2010.07.081 only first-line therapeutic option. However, the mortality was still high, ${ }^{9,10}$ reaching $20 \%$ within 1 month for type A dissection and $10 \%$ for type B dissection. This mortality rate resulted from persistent malperfusion syndrome or aortic rupture. Spiral multidetector computed tomography (CT) angiography ${ }^{11-13}$ can be used to examine the aorta from the thoracic segment up to the femoral arteries. High enhancement with adequate spatial resolution has made it possible to analyze the branches of the aorta. A new CT-based classification system has been developed to describe the mechanism underlying malperfusion. ${ }^{14,15}$ With better analysis of the entire aorta and because of all the complications resulting from aortic dissection, the management strategy was adapted, ${ }^{16,17}$ and endovascular treatment became an option. A choice was made among thoracic stent grafting, peripheral stenting, or fenestration, depending on the mechanism and complications resulting from aortic dissection. This complex disease required multidisciplinary input in both the emergency phase and during follow-up, with formalized cooperation among cardiac surgeons, vascular surgeons, cardiologists, anesthesiologists, and interventional radiologists.

The present study evaluated the results of endovascular fenestration in a selected population of patients with aortic dissection complicated by malperfusion syndrome due to dynamic compression. The focus was on the efficacy in the emergency phase and the long-term outcomes. 


\section{Abbreviation and Acronym \\ $\mathrm{CT}=$ computed tomography}

\section{MATERIALS AND METHODS}

Between December 1999 and November 2007, 728 patients from a 4-million population area were referred to our university hospital for type A $(n=376)$ or type $B(n=352)$ aortic dissection. Malperfusion symptoms were present in 145 of the 728 patients. These 145 patients were treated by thoracic and peripheral stenting in 110 and fenestration in 35 . The risk factors and clinical symptoms of the global population (age range 39-77 years) are listed in Table 1. Ischemic bowel disease $(n=27)$ was suspected in patients with abdominal pain, digestive tract bleeding, diarrhea, and/or abnormal test results (high lactate levels and elevations of creatine phosphokinase, lipase, amylase, and transaminase activity). Renal ischemia $(n=27)$ was investigated in patients with anuria and/or an elevated creatinine increase coupled with lower limb ischemia $(n=14)$ (pain and coldness in the leg). Two patients were paraplegic.

A CT scan confirmed that the true lumen was under compression by the false lumen (dynamic compression) in all cases at the level of the visceral aorta, with or without extension of the dissection into the visceral arteries. Several territories could be involved in a single patient.

Of the 35 patients treated with fenestration, 19 presented with type A dissection. Cardiac complications were present, and cardiac surgery was performed immediately in 10 of the 19 patients. Because of severe bowel ischemia, first-line fenestration was performed in 9 of 19 patients. The cardiac surgery involved ascending aorta replacement in 16 patients (Bentall in 14 patients and Tyron-David in 2 patients) and was completed by hemiarch replacement in 7 patients. Aortic valve replacement was necessary in the case of a valve lesion from aortic dissection or in the case of degenerative disease or Marfan syndrome. Aortic valve replacement was not performed systematically. Three patients with type A dissection died before surgical repair ( 2 of strokes and 1 of bowel ischemia owing to late arrival at the hospital).

The mean interval between the onset of chest pain and endovascular fenestration was 4 days (range, 4 hours to 41 days). Most patients (28/35) were treated within 3 days. For the 7 remaining patients, delayed treatment resulted from a late diagnosis $(\mathrm{n}=5)$ or a poor outcome after peripheral stenting $(\mathrm{n}=1)$ or vascular surgery alone $(\mathrm{n}=1)$. One patient had undergone peripheral grafting (femoro-femoral bypass) for lower limb ischemia

TABLE 1. Clinical characteristics of total population $(n=35)$

\begin{tabular}{lc}
\hline \multicolumn{1}{c}{ Characteristic } & Value \\
\hline Sex & \\
$\quad$ Male & 31 \\
Female & 4 \\
Mean age (y) & $57 \pm 11$ \\
Type A aortic dissection (n) & 19 \\
Type B aortic dissection (n) & 16 \\
Marfan, ectasia disease (n) & 4 \\
Hypertensive (n) & 22 \\
Tobacco use (n) & 15 \\
Symptoms (n) & \\
Bowel ischemia & 27 \\
Renal ischemia & 27 \\
Lower limb ischemia & 14 \\
Paraplegia & 2 \\
\hline
\end{tabular}

due to aortic dissection but was then referred for fenestration because of residual visceral ischemia.

\section{Fenestration Technique}

The decision for the treatment of aortic dissection was made by the cardiac surgeon, depending on the symptoms of the patient, biologic parameters, CT scan imaging analysis, and after discussion with the cardiologists from the intensive care unit and interventional radiologists. Surgery was immediately performed for type A aortic dissection with cardiac complications or without malperfusion symptoms. When malperfusion symptoms were present, specifically bowel ischemia, endovascular treatment was performed first. Fenestration was performed if persistent symptoms of ischemia and evidence of a dynamic mechanism causing the malperfusion were present. Before the intervention, all patients underwent $\mathrm{CT}$ angiography from the thoracic to the femoral segments with contrast enhancement.

The fenestration procedure has been previously described. ${ }^{18}$ In brief, 2 rigid guidewires (Amplatz or Superstiff; Boston Scientific, Nanterre, France) were introduced in a $8 \mathrm{~F}$ sheath (straight introducer sheath, $45 \mathrm{~cm}$ in length; Arrow International, Diegem, Belgium) by a trained interventional radiologist. A femoral approach route was used in all patients, either right (32 patients) or left (3 patients), sometimes completed using a humeral approach if difficulties were encountered ( 2 patients). The distal end of the introducer sheath was positioned into the external iliac artery. Usually the first guidewire was pushed directly inside the true lumen. To catheterize the false lumen, a preshaped catheter (RDC 4F; Cordis, Johnson \& Johnson, Warren/Bridgewater, NJ) with a hydrophilic guide wire (preshaped, 0.035in, 150-cm length; Terumo, Somerset, NJ) was inserted into the same introducer. The communication was assessed before the procedure using CT and was usually located at the level of the iliac bifurcation (external and hypogastric arteries). No septum puncture was done. When the 2 guidewires were in both lumen, the introducer sheath was advanced from the external iliac artery up to the visceral arteries to destroy the intimal flap. Next, after fenestration, and to expand the true lumen, a large balloon (diameter of $20 \mathrm{~mm}$ or more; Cordis, Johnson \& Johnson) was inflated inside the true lumen at the level of the descending aorta and pulled back, still inflated, down to the iliac bifurcation. The guidewire inside the false lumen was withdrawn for this maneuver. Angiography was then performed inside the true lumen. Angiographic success was defined as the ability on the angiogram to visualize the true and false lumen into the aorta with rapid enhancement at the same time and with large entry tears. If visualization of the visceral arteries was not adequate, peripheral stenting was performed (Video 1).

Most patients (26/35) underwent additional endovascular procedures, either concurrently or within a few days. Peripheral stenting was performed if dissection extension was present to the collateral arteries with malperfusion or if the fenestration had been insufficient. The collateral arteries concerned were the renal arteries in 14 , the superior mesenteric artery in 5, the celiac artery in 4 , and the iliac arteries in 9 . Two patients had a stent implanted in the subrenal aorta because of postfenestration, residual, lower limb ischemia. This aortic stenting was necessary, because entry tears were present between the true and false lumen close to the renal arteries. After fenestration, projection of the intimal flap into the abdominal aorta was responsible for lower limb ischemia, which was successfully treated by additional abdominal bare metal stenting (Palmaz P4014 or P5014; Cordis). One patient had a thoracic stent graft (38 $\mathrm{mm}$ in diameter, Talent; Medtronic, New York, NY) to close the proximal entry gate, because of postfenestration, residual renal ischemia. The patients were evaluated using CT or magnetic resonance imaging and were regularly examined every 6 or 12 months. The follow-up period was defined as the interval between aortic dissection and the last imaging procedure performed. The largest aortic diameter was measured in a plane orthogonal to the main axis of the aorta. The measurements were performed in both the thoracic and the abdominal segments. The comparison of the aortic diameters before fenestration and during follow-up was performed using the Wilcoxon signed-rank test. 
TABLE 2. Parameters of endovascular fenestration technique and early and late (mean follow-up, 3 years) results in global population

\begin{tabular}{lc}
\multicolumn{1}{c}{ Outcome } & Value \\
\hline Technical success rate (\%) & 100 \\
Additional endovascular intervention (n) & $26 / 35$ \\
Secondary endovascular interventions (n) & 22 \\
Renal stenting (n) (stents, patients) & 26,14 \\
Superior mesenteric artery and celiac trunk (n) & 9,9 \\
$\quad$ (stents, patients) & \\
Lower limb arteries (n) (stents, patients) & 15,9 \\
Abdominal stents (n) (stents, patients) & 6,5 \\
Thoracic stent graft (n) (stents, patients) & 1,1 \\
Additional fenestration (n) & 6 \\
Additional surgical intervention (n) (patients) & 9 \\
Bowel surgery (n) & 7 \\
Additional cardiac surgery (n) & 1 \\
Aponeurectomy (n) & 1 \\
Angiographic success & $34 / 35(97 \%)$ \\
Mortality at 1 mo & $12 / 35$ \\
Global mortality (mean follow-up 3 y) & $16 / 35$ \\
Initial mean diameter of thoracic aorta (mm) & $38 \pm 8$ \\
Initial mean diameter of abdominal aorta (mm) & $32 \pm 7$ \\
Mean diameter of thoracic aorta at follow-up (mm) & $45 \pm 11$ \\
Mean diameter of abdominal aorta at follow-up (mm) & $36 \pm 10$ \\
\hline
\end{tabular}

\section{RESULTS}

The main early and late results for the global population are summarized in Table 2. Fenestration was technically feasible in all patients $(100 \%$ technical success rate). In patients with calcifications on the intimal flap or if repair of the aortic dissection had been delayed, rupture of the intimal flap needs to push hardly the introducer sheath to advance the sheath on the guidewires. No complicationsaortic or iliac rupture-were observed after fenestration. The fenestration relieved the compression of the true lumen as assessed by angiography in 34 of the 35 patients (angiographic success rate, $97 \%$ ). Additional stenting was necessary when the dissection had extended into the visceral or lower limb arteries. If the fenestration was extended up to the distal end of the dissection, the intimal flap below was repaired using a balloon-expanded stent to prevent ischemia. The intimal flap at the level of fenestration and above was left in place and did not ever result in embolization or ischemia. An example of 1 patient with bowel, renal, and lower limb ischemia, treated by fenestration and additional right renal stenting, is shown in Figures 1 and 2.

Within 1 month, 12 (34\%) of the 35 patients had died (Figure 3). Of the 19 patients in the type A aortic dissection group, $8(42 \%)$ had died, either after cardiac surgery (5 patients) or before surgery ( 3 patients because of stroke in 2 and bowel ischemia owing to late arrival at the hospital in 1). The reasons for death were cardiac and/or cerebral complications (hemopericardium, acute aortic insufficiency, stroke) or severe malperfusion syndrome. Of the
16 patients in the type B dissection group, $4(25 \%)$ had died of multiple organ failure secondary to ischemia. Of the 7 patients who underwent emergency surgery for mesenteric necrosis, 3 died.

Of the 23 survivors, recovery from fenestration was slow in 8 patients because of ischemic complications in the first month, including renal, digestive, hemodynamic, and neurologic complications. Necrotic intestinal complications in 4 patients were treated with surgical bowel resection during follow-up. The renal complications include acute kidney failure in 2 patients, both of whom had already had chronic renal failure. Temporary dialysis was required. Two patients developed a hematoma at the puncture point, with satisfactory resolution without transfusion. One patient developed a hemothorax that was treated by drainage, and one patient developed multiple organ failure, with a satisfactory outcome after intensive care. Finally, 1 patient had a transient ischemic accident due to a cardiac embolism.

At follow-up, 4 patients had died without any information available (range, 4 months to 7 years; mean, more than 3 years), and 2 patients had withdrawn from the study. Of the 17 survivors, 6 had had late-stage complications. Two patients with type B dissection had developed secondary retrograde dissection, and replacement of the ascending aorta was necessary. Two patients developed secondary extension of the dissection into the collateral arteries and were treated by peripheral stenting. One patient had unilateral renal atrophy due to chronic renal ischemia, and another developed residual mesenteric angina due to secondary obstruction of the stent in the mesenteric artery.

At a mean follow-up of $48 \pm 30$ months, the diameter of the main thoracic aorta was $45 \pm 11$ (range, 30-60 mm) compared with the initial $38 \pm 8 \mathrm{~mm}(P=\mathrm{NS})$. No change in the aortic diameter was observed in 12 patients with a patent false lumen. Five patients had an increased aortic diameter (multiple arterial ectasia in 4 patients and Marfan syndrome in 1). All false lumen were patent without any thrombosis, either partial or complete. The diameter of the abdominal aorta at follow-up was $36 \pm 10 \mathrm{~mm}$ (range, 26-57 mm) compared with the initial $32 \pm 7 \mathrm{~mm}$ $(P=\mathrm{NS})$. Additional procedures performed during the follow-up period included 3 successful thoracic stent grafts in 2 patients to repair aneurysms in the descending aorta and 1 surgical procedure to repair an abdominal aneurysm.

\section{DISCUSSION}

The present study has demonstrated that endovascular fenestration is safe and effective in the treatment of dynamic compression at the origin of visceral or peripheral malperfusion in the presence of complicated aortic dissection. Furthermore, after fenestration, the diameter of the aorta did not enlarge in the long term.

The fenestration procedure was easy to perform, with a $100 \%$ technical success rate. The technique was 

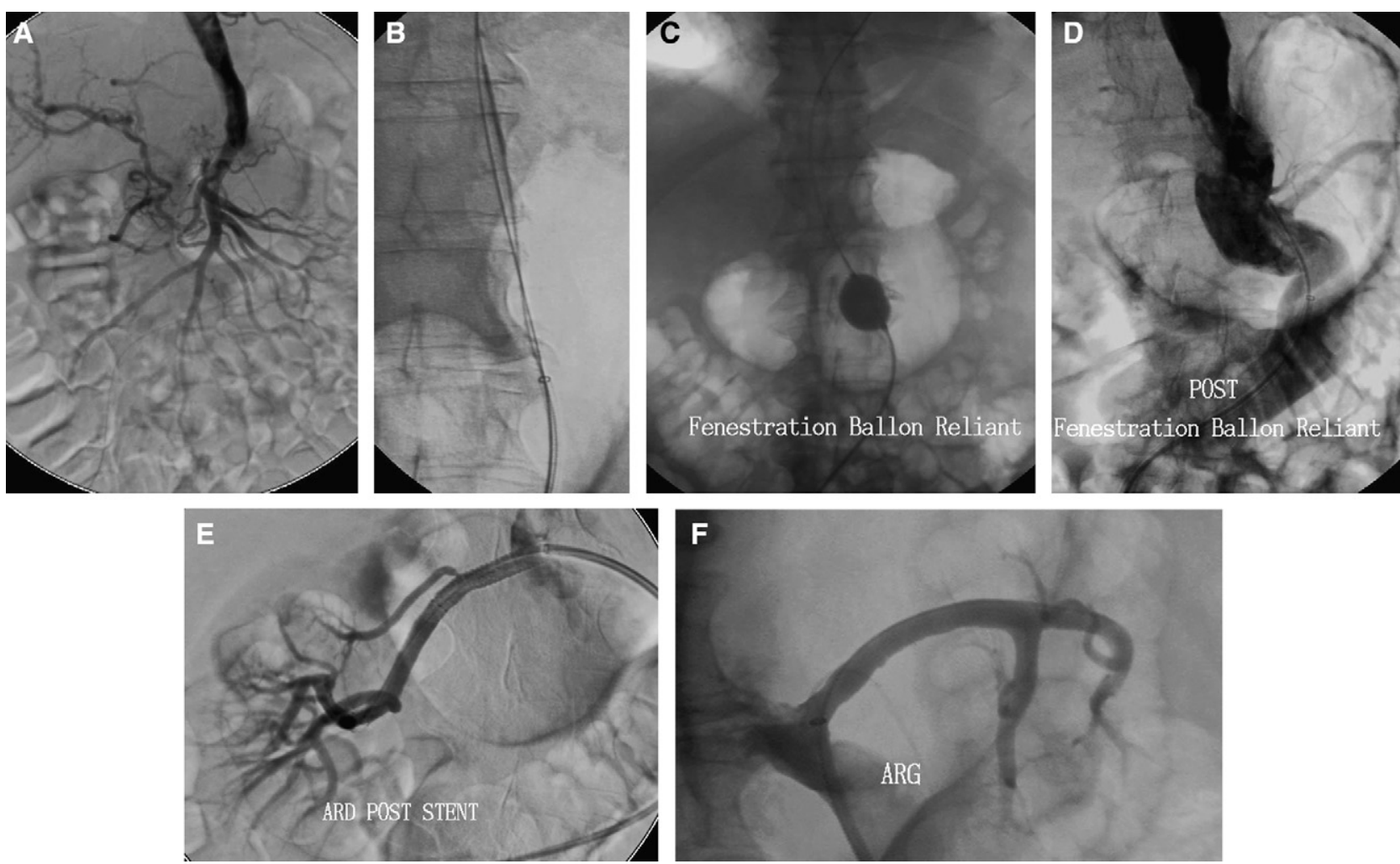

FIGURE 1. Angiogram into true lumen before fenestration (A) showing virtual true lumen with opacification of celiac trunk and superior mesenteric artery. Abdominal aorta and renal arteries were not visualized by injection of contrast media into descending thoracic aorta. B, Fenestration technique with 2 guidewires into 1 introducer sheath starting at level of right iliac artery up to celiac trunk. C, A large balloon was then inflated into the true lumen and pulled down from the thoracic aorta to the abdominal aorta. D, After fenestration, angiography of bowel arteries and abdominal aorta was obtained. E, Acute intrarenal dissection $(A R D)$ treated with 2 self-expandable stent inducing complete recovery of right kidney perfusion. F, Selective arteriogram performed on left renal artery $(A R G)$ showed no need for additional treatment.

performed by 5 operators trained in endovascular techniques. The femoral puncture site was chosen by reading the CT scan to ensure catheterization of both lumen as distally as possible (usually at the iliac level). In almost all cases, the first guidewire went into the true lumen. To catheterize the false lumen, a preshaped catheter with a hydrophilic guidewire was used. The level of re-entry, suspected on the CT scan, was confirmed by angiography performed through the introducer inserted by way of the common femoral artery and reaching the external iliac artery. Resistance to the advance of the introducer owing to the intimal flap was observed when the hydrophilic guidewire was used to catheterize the false lumen. The presence of calcification opposed the advance of the introducer and compromised flap destruction. At the beginning of our series, fenestration was performed up to the level of the visceral arteries; however, after 12 cases, the length of the fenestration was reduced. The thought was just to open the entry tear between the true and false lumen to relieve the compression. By creating a minimal communication (3-5 cm long), the hope was to repair the aortic dissection and at a later stage to close all entry tears. However, this technique failed to correct the malperfusion syndrome ( 5 patients) and open the true lumen, even with inflation of a large balloon inside from the thoracic aorta down to the abdominal aorta. Our explanation was the presence of a Venturi effect inside the true lumen, pulling the intimal flap in front of the visceral arteries coming from the true lumen. In these cases, extension of the fenestration was necessary. The fenestration procedures in the remaining patients were extended up to the visceral arteries. In addition, a large balloon $(>18 \mathrm{~mm}$ in diameter), inflated inside the true lumen, was pulled down from the thoracic aorta to the abdominal aorta. After fenestration, arteriography of the visceral and peripheral arteries indicated the need for peripheral stenting. When fenestration was not started at the distal end of the dissection but above, the risk was that the intimal flap below the fenestration would induce peripheral ischemia. In fact, the distal flap was not maintained in place and fell down over the aorto-iliac bifurcation. Stenting inside the true lumen was obligatory in this situation, whether in the abdominal aorta or the iliac arteries. In our experience, balloon-expanded stents are preferable to self-expanded stents (which will be compressed by the false lumen). 

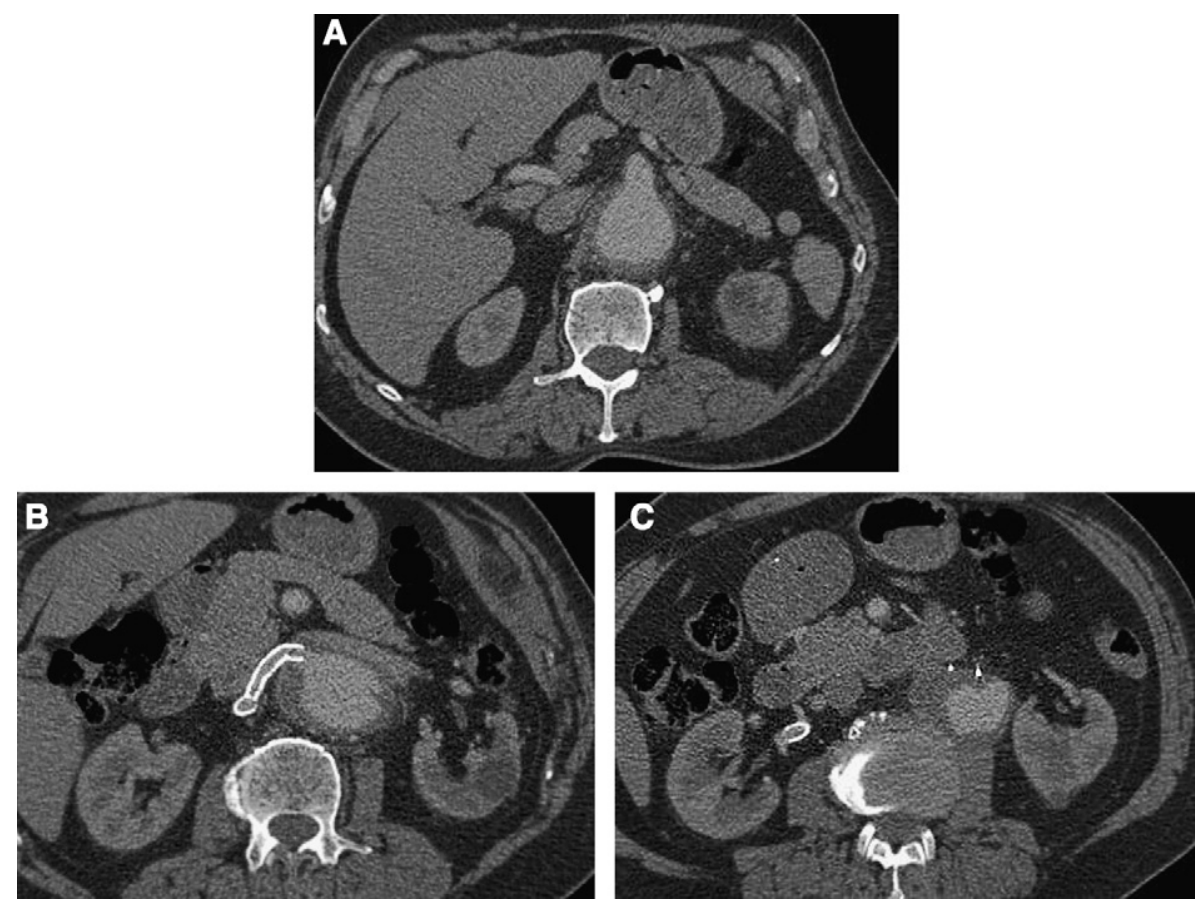

FIGURE 2. Computed tomography $(C T)$ angiogram at 2 years of follow-up demonstrated good efficacy of fenestration with good patency of superior mesenteric artery (A) and good patency of proximal (B) and distal (C) stenting of right renal artery. Both kidneys were enhanced at same time.

Other techniques of fenestration are possible. ${ }^{14,19,20}$ The advantages of our technique include the cost, effectiveness, and rapidity of symptom relief. The indications for fenestration were determined by the clinical symptoms, abnormal laboratory test results, and analysis of the CT scan. Abdominal pain, lactate increase, anuria, and creatinine increase were the most frequent signs pointing to malperfusion. Lower leg ischemia might also be a consequence of malperfusion due to dynamic compression. The CT scan was the main examination used to analyze the complete aorta and its branches. If the CT scan showed compression of the true lumen by the

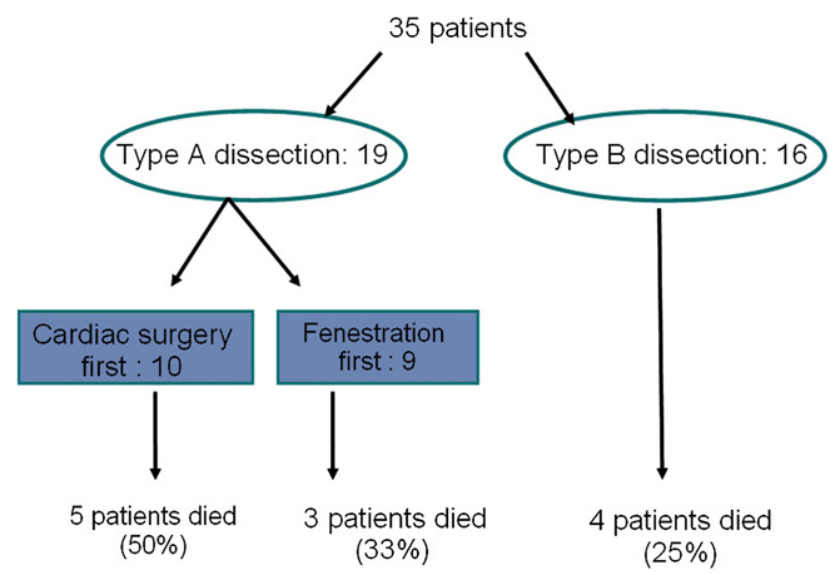

FIGURE 3. Early mortality in population depending on type of dissection and moment of fenestration. false lumen in front of the visceral arteries, dynamic compression was causing the malperfusion. ${ }^{12,13}$ The main explanation is the presence of a large proximal entry tear and a small distal entry tear. The ideal treatment is to close the proximal entry tear, either surgically or using a thoracic stent graft. ${ }^{21-23}$ Surgery should be performed in the case of type A dissection. However, compression of the true lumen is not always obtained after surgery because of the impossibility of closing all the main proximal entry tears. Some of these could be located in the arch or in the descending thoracic aorta. A thoracic stent graft is a good option in the case of type B dissection, although this technique requires special equipment, roundthe-clock presence of trained operators, and an accurate evaluation of the device size. Also, implanting a thoracic stent graft in an acute dissection risks the occurrence of complications such as retrograde dissection because the aorta is fragile owing to recent rupture. ${ }^{24}$ Finally, emergency thoracic stent grafting was not possible at our center at the beginning of the present series. Multidisciplinary discussions with cardiac and vascular surgeons, cardiologists, anesthesiologists, and interventional radiologists were always organized to determine the best treatment. If a type A aortic dissection was present with cardiac complications and malperfusion syndrome, heart surgery was performed first. Endovascular treatment was scheduled if symptoms of malperfusion persisted. If type A aortic dissection occurred with evidence of visceral malperfusion but no cardiac complications, endovascular treatment was performed 
before surgery. ${ }^{16,17}$ In type B aortic dissection complicated by malperfusion, fenestration was performed if the symptoms were acute. This technique was fast and could be done as an emergency procedure by all physicians $(\mathrm{n}=6)$, both day and night.

Analysis of the CT scan was critical to the localization of the dissection in the thoracic aorta; the true and false lumen; the compression of the true lumen in the aorta; extension of the dissection into the branches; and ischemia of the bowel tract and/or kidneys and/or lower leg. This analysis influenced the management strategy and the choice of medical, surgical, or endovascular treatment. All patients with malperfusion syndrome were receiving whole heparin to manage the ischemia. This treatment was stopped 48 hours after treatment of the malperfusion and was substituted with a preventive dose of heparin. $\beta$-Blockers and angiotensin-2 receptor antagonist or angiotensin-converting enzyme inhibitors were the main drugs used. One of the main objectives was to maintain the systolic pressure from 130 to $100 \mathrm{~mm} \mathrm{Hg}$. Refractory hypertension was mainly due to persistent malperfusion owing to static compression. Secondary stenting of branches was necessary in many cases. Also, bowel surgery was necessary to excise necrotic tissue. In our experience, such necrosis developed after severe ischemia lasting more than 6 hours. Dialysis was necessary in the case of persistent renal failure.

The other major finding of the present study concerned the changes in the overall diameter of the aorta. After a mean follow-up of 3 years, $70 \%$ of the patients had had no significant increase in the diameter of the aorta. This result was in accordance with the fact that fenestration decreased the load and pressure inside the false lumen by increasing communication between the 2 lumen. No partial thrombosis occurred in the false lumen (which has been found to increase the 3 -year mortality rate ${ }^{25}$ ). With fenestration performed in the early phase of aortic dissection with acute complications, no side effects were recorded during follow-up. Because aortic dissection was still present, follow-up examinations were necessary to check for enlargement of the aorta and aortic wall disease (for which the only treatment is surgical replacement of the entire aorta). The purpose of endovascular treatment is to save the patient with acute complications with a view to administering optimum treatment-surgery or medical treatmentin the chronic phase. Aortic dissection has become a chronic disease that we must learn to manage while the patient is still alive.

The major limitation of the present study was that patients with complicated aortic dissection were treated using a specific method at a single center. The choice of endovascular fenestration was determined from the CT scan analysis and acute symptoms; all decisions were discussed by the multidisciplinary team. A randomized study comparing fenestration and thoracic stent grafting would be interesting; however, because of the small number of patients and the emergency status such a trial would not be possible. Another limitation was the diversity of the patients included in the present study. Every case was different in terms of complications and the morphology of the dissection. The strong point of the present study was the grouping of 35 patients with aortic dissection and acute complications, although nevertheless a small population. We must learn more about the management of aortic dissection before the fenestration technique can be proposed for all our patients. However, the results of the present study have demonstrated the safety of the technique and shown promising results in the early phase of acute complications and good long-term outcomes.

\section{References}

1. Sans S, Kesteloot H, Kromhout D. Task Force. Task Force of the European Society of Cardiology on cardiovascular mortality and morbidity statistics: Europe. Eur Heart J. 1997;18:1231-48.

2. Taylor KM. Diseases of the aorta. In: Julian DG, Camm AJ, Fox KM, Hall RJC, Poole-Wilson PA, editors. Diseases of the heart. London: Bailliere Tindall; 1989. p. 1338-62.

3. Roberts WC. Aortic dissection: Anatomy, consequences and causes. Am Heart J. 1981;101:195-214

4. Cambria RP, Brewster DC, Gertler J, Moncure AC, Gusberg R, Tilson MD, et al Vascular complications associated with spontaneous aortic dissection. $J$ Vasc Surg. 1988;7:199-209.

5. McDonald GR, Schaff HV, Pyeritz RE, McKusick VA, Gott VL. Surgical management of patients with the Marfan syndrome and dilatation of the ascending aorta. J Thorac Cardiovasc Surg. 1981;81:108-15.

6. Crawford ES, Svensson LG, Coselli JS, Safi HJ, Hess KR. Surgical treatment of aneurysm and/or dissection of the ascending aorta, transverse aortic arch: Factors influencing survival in 717 patients. J Thorac Cardiovasc Surg. 1989;98:659-74.

7. Borst HG, Jurmann M, Buhner B, Laas J. Risk of replacement of descending aorta with a standardized left heart bypass technique. J Thorac Cardiovasc Surg. 1994;107:126-33.

8. McDonald GR, Schaff HV, McKusick VA, Gott VL, Pyeritz RE. Surgical management of patients with the Marfan syndrome and dilatation of the ascending aorta. J Thorac Cardiovasc Surg. 1981;81:180-6.

9. Hagan PG, Nienaber CA, Isselbacher EM, Bruckman D, Karavite DJ, Russman PL, et al. The international registry of acute aortic dissection (IRAD): New insights into an old disease. JAMA. 2000;283:897-903.

10. Trimarchi S, Nienaber CA, Rampoldi V, Myrmel T, Suzuki T, Mehta RH, et al. International Registry of Acute Aortic Dissection Investigators. Contemporary results of surgery in acute type A aortic dissection: The International Registry of Acute Aortic Dissection experience. J Thorac Cardiovasc Surg. 2005;129: 112-22.

11. Nienaber CA, von Kodolitsch Y, Nicolas V, Siglow V, Piepho A, Brockhoff C, et al. The diagnosis of thoracic aortic dissection by noninvasive imaging procedures. N Engl J Med. 1993;328:1-9.

12. Williams DM, Lee DY, Hamilton BH, Marx MV, Narasimham DL, Kazanjian SN, et al. The dissected aorta: Part III. Anatomy and radiologic diagnosis of branch-vessel compromise. Radiology. 1997;203:37-44.

13. Sommer T, Fehske W, Holzknecht N, Smekal AV, Keller E, Lutterbey G, et al Aortic dissection: A comparative study of diagnosis with spiral CT, multiplanar transesophageal echocardiography, and MR imaging. Radiology. 1996;199: 347-52.

14. Slonim SM, Miller DC, Mitchell RS, Semba CP, Razavi MK, Dake MD. Percutaneous balloon fenestration and stenting for life-threatening ischemic complications in patients with acute aortic dissection. J Thorac Cardiovasc Surg. 1999 117:1118-27.

15. Gaxotte V, Cocheteux B, Haulon S, Vincentelli A, Lions Ch, Koussa M, et al. Relationship of intimal flap position to endovascular treatment of malperfusion syndromes in aortic dissection. J Endovasc Ther. 2003;10:719-27. 
16. Fabre O, Vincentelli A, Willoteaux S, Beregi J-P, Prat A. Preoperative fenestration for type A acute aortic dissection with mesenteric malperfusion. Ann Thorac Surg. 2002;73:950-1.

17. Deeb GM, Williams DM, Bolling SF, Quint LE, Monaghan H, Sievers J, et al. Surgical delay for acute type A dissection with malperfusion. Ann Thorac Surg. 1997;64:1669-75.

18. Beregi JP, Prat A, Gaxotte V, Delomez M, McFadden EP. Endovascular treatment for dissection of the descending aorta. Lancet. 2000;356:482-3.

19. Chavan A, Hausmann D, Dresler C, Rosenthal H, Jaeger K, Haverich A, et al. Intravascular ultrasound-guided percutaneous fenestration of the intimal flap in the dissected aorta. Circulation. 1997;96:2124-7.

20. Elefteriades JA, Hammond JL, Gusberg RJ, Kopf GS, Baldwin JC. Fenestration revisited: A safe and effective procedure for descending aortic dissection. Arch Surg. 1990;125:786-90

21. Dake MD, Kato N, Mitchell RS, Semba CP, Razavi MK, Shimono T, et al. Endovascular stent-graft placement for the treatment of acute aortic dissection. $N$ Engl J Med. 1999;340:1546-52.
22. Nienaber CA, Fattori R, Lund G, Dieckmann C, Wolf W, von Kodolitsch Y, et al. Non surgical reconstruction of thoracic aortic dissection by stent-graft placement. N Engl J Med. 1999;340:1539-45.

23. Fattori R, Nienaber CA, Rousseau H, Beregi J-P, Heijmen R, Grabenwoger M, et al. Talent thoracic retrospective registry: Results of endovascular repair of the thoracic aorta with the Talent Thoracic stent graft-The Talent Thoracic Retrospective Registry. J Thorac Cardiovasc Surg. 2006;132: 332-9.

24. Eggebrecht H, Thompson M, Rousseau H, Czerny M, Lönn L, Mehta RH, et al. European Registry on Endovascular Aortic Repair Complications. Retrograde ascending aortic dissection during or after thoracic aortic stent graft placement: Insight from the European registry on endovascular aortic repair complications. Circulation. 2009;120:S276-81.

25. Tsai TT, Evangelista A, Nienaber CA, Myrmel T, Meinhardt G, Cooper JV, et al. International Registry of Acute Aortic Dissection. Partial thrombosis of the false lumen in patients with acute type B aortic dissection. N Engl J Med. 2007;357: 349-59. 